\title{
Gallbladder interstitial Cajal-like cells and gallbladder contractility in patients with cholelithiasis: a prospective study
}

\author{
Runyu Ding, Junmin Wei, Jingyong Xu
}

National Center of Gerontology, Beijing Hospital, Beijing, China

\begin{abstract}
Introduction. A reduced number of interstitial Cajal-like cells (ICLCs) in the gallbladder have been proposed to play a role in the pathogenesis of cholelithiasis. Therefore, this prospective study was conducted to investigate the relationship between gallbladder contractility and the number of gallbladder ICLCs in patients with cholelithiasis. Material and methods. Patients admitted to the Department of Hepatobiliary Surgery for cholecystectomy were divided into the cholelithiasis $(\mathrm{n}=18)$ and non-cholelithiasis $(\mathrm{n}=8)$ groups based on their clinical data. Patients' clinical data were collected on admission, and B-mode ultrasonography was performed to assess their gallbladder contractility. The resected gallbladder specimens were fixed, paraffin sections mounted on slides, and the immunofluorescence staining with the anti-human CD-117 and anti-human tryptase antibodies was performed to identify ICLSs and mast cells, respectively. The number of ICLCs was counted in 10 high-power fields (HPFs) randomly.

Results. Independent sample t-tests revealed differences between the cholelithiasis and non-cholelithiasis groups in the number of ICLCs (mean \pm standard deviation: $88.61 \pm 28.22$ vs. $115.89 \pm 27.87$ per HPFs, $\mathrm{P}=0.032$ ) and gallbladder contractility $(43.94 \% \pm 18.50 \%$ vs. $61.00 \% \pm 20.50 \%, \mathrm{P}=0.046)$. Pearson and Spearman correlation analyses revealed no significant correlation between the number of ICLCs and gallbladder contractility. Conclusion. The results suggest that the number of gallbladder ICLCs in the wall of the gallbladder of patients with or without cholelithiasis is not a decisive factor affecting gallbladder contractility. (Folia Histochemica et Cytobiologica 2019, Vol. 57, No. 2, 94-100)
\end{abstract}

Key words: cholelithiasis; interstitial Cajal-like cells; mast cells; gallbladder contractility; immunofluorescence; ultrasonography

\section{Introduction}

Cholelithiasis combined with chronic cholecystitis is a common disease that often requires hepatobiliary surgery, and its incidence in developing countries is approximately $10-15 \%$, with a higher prevalence in women [1]. The pathogenesis of gallstones is considered to be associated with bile cholesterol supersaturation, abnormalities in cholesterol nucleation, and impaired gallbladder function, which is believed to

\footnotetext{
Correspondence addres: Jingyong $\mathrm{Xu}$

National Center of Gerontology,

Beijing Hospital, No. 1 Dahua Road,

Dongdan, Dongcheng District, Beijing, 100730

phone: 13810351797; fax: 85136262

e-mail: bjhxujingyong@163.com
}

be of great significance. Interstitial Cajal-like cells (ICLCs) are thought to be associated with the spontaneous rhythmic movements of the gastrointestinal tract [2], and they are considered the pacemaker cells of slow-wave potential in gastrointestinal smooth muscles. The typical ICLCs are elongated, fusiform bodies with few processes. Large ones have an oval nucleus. Numerous mitochondria can be observed in the cytoplasm under an electron microscope; they express the c-kit receptor, which can be identified by CD117 (c-kit protein) [3]. These cells also exist in the gallbladder wall in many species, including humans and guinea pigs. It was found that ICLCs are closely related to gallbladder function in guinea pigs [4]. In recent years, studies conducted in China and abroad have shown that the density of ICLCs was significantly lower in the gallbladder wall of patients with cholelith- 
iasis than of those without cholelithiasis [5]. Hence, it was proposed that the reduced number of ICLCs may play a role in the pathogenesis of cholelithiasis.

The aim of this prospective study was to investigate the relationship between gallbladder contractility and the number of ICLCs in the gallbladder wall of patients with cholelithiasis.

\section{Material and methods}

Ethical approval. The study was conducted in accordance with the moral, ethical, regulatory, and scientific principles governing clinical research. All surgical samples were retrieved with the approval of the Beijing Hospital ethical Committee the Declaration of Helsinki (protocol number 2016BJYYEC-057-01) and oral informed consent was obtained from the patients.

Subjects. The subjects enrolled in this study were patients admitted to the Department of Hepatobiliary Surgery in our hospital between December 2016 and March 2017, who required cholecystectomy owing to cholelithiasis combined with chronic cholecystitis or other non-cholelithiasis reasons. The patients were divided into the cholelithiasis and non-cholelithiasis groups, according to their clinical characteristics.

Inclusion and exclusion criteria. Inclusion criteria for the cholelithiasis group were as follows: 1) patients with cholelithiasis with clear indications for surgery and no obvious contraindications; and 2) patients with no history of acute cholecystitis in the past 4 weeks.

Inclusion criteria for the non-cholelithiasis group were as follows: 1) patients with no history of cholelithiasis and cholecystitis (i.e., no gallstones were detected during preoperative imaging); and 2) patients who required cholecystectomy because of gallbladder polyps, gallbladder adenomyomatosis, or other reasons.

Exclusion criteria for both groups were as follows: 1) patients with acute cholecystitis or a history of acute cholecystitis in the past 4 weeks; 2) patients with a history of biliary system surgery, such as endoscopic retrograde cholangiopancreatography and endoscopic sphincterotomy; 3) patients with cholelithiasis combined with gallbladder polyps or gallbladder adenomyomatosis; 4) patients with the presence of obstructive jaundice, or intrahepatic or extrahepatic biliary stones; and 5) patients with a history of gastrointestinal anastomosis and other surgical changes to gastrointestinal continuity.

Materials and instrumentation. The following materials and instruments were used: the ProSound-Alpha 7 Color Ultrasound Diagnostic System (Hitachi Aloka Medical, Tokyo, Japan), Nikon CI-S Microscope, Nikon DS-U3 Imaging System, Image-Pro Plus 6.0 Image Analysis Software
(MediaCybernetics, Rockville, MD, USA), xylene, ethylenediamine-tetraacetic acid (EDTA) retrieval solution, rabbit anti-human CD-117 antibody (ab5505, Abcam, Cambridge, UK), mouse anti-human tryptase antibody (ab2378, Abcam), phosphate buffer solution (C1014, Jiamei Biotech, Co., Ltd, Guangzhou, China), bovine serum albumin fraction $\mathrm{V}$ (A8020, Solarbio, Beijing, China), Cy3-labeled goat anti-rabbit secondary antibody (ab97075, Abcam), biotin-labeled goat anti-mouse secondary antibody (ab150113, Abcam), and 4',6-diamidino-2-phenylindole (DAPI) color-developing agent (CH126, Jiamay).

Determination of gallbladder contractility. Patients' medical history was obtained on admission, and they were assigned to one of the two groups according to the aforementioned criteria. Patients underwent a fasting B-mode ultrasonography examination on the morning after admission. The examination was conducted in the supine or left lateral decubitus position. The abdominal probe was used to detect the gallbladder along the right subcostal margin. A freeze-frame image was taken at the maximum cross-section of the gallbladder, and its maximum length and width were measured. Then, the probe was rotated clockwise by $90^{\circ}$, and another freeze-frame image was taken to measure the height of the gallbladder. After recording the aforementioned data, the patients were instructed to quickly consume some yogurt and wait for $45 \mathrm{~min}$ before the length, width, and height of the gallbladder were measured again using the same methods. The fasting and post-prandial B-mode ultrasonography examinations were conducted in the same position for each patient. The volume of the gallbladder was calculated using the ellipsoid volume formula. The gallbladder contraction rate was calculated based on the following formula: 1-(45-minute gallbladder volume/fasting gallbladder volume) $\times 100 \%$. The fatty-meal B-mode ultrasonography examination for all patients was conducted by the same physician.

Sample collection and immunohistochemistry. Patients underwent elective cholecystectomy after completing the preoperative preparations (including but not limited to chest radiography, electrocardiography, and routine blood tests to ensure safety). Intraoperatively, the degree of pericholecystic adhesions and the presence of abnormalities in the cystic artery and cystic duct were recorded. All resected gallbladder specimens were immersed in a $4 \%$ formaldehyde solution and sent to the pathology department. One tissue section $(0.2 \times 0.5 \times 0.8 \mathrm{~cm})$ was obtained from the neck, body, and fundus of each gallbladder specimen.

The sections were embedded in the same paraffin block by standard histological technique, and cut into slides for immunofluorescence staining. Next, the slides were immersed in xylene three times for $10 \mathrm{~min}$ each, followed sequentially by immersion in $100 \%, 95 \%$, and $80 \%$ ethanol for $10 \mathrm{~min}$ each, and then rinsed thoroughly. EDTA retrieval solution was added, and the slides were heated in the microwave 
(101-1BS, Bangxi Corp., Shanghai, China) at $65^{\circ} \mathrm{C}$ for $30 \mathrm{~min}$ to restore the antigenicity of the specimens. After rinsing, 3\% hydrogen peroxide (prepared with deionized water) was added, and the sections were incubated at room temperature (RT) for $30 \mathrm{~min}$ and rinsed three times with phosphate-buffered saline (PBS) at RT for 5 min each. A working solution of bovine serum albumin fraction $\mathrm{V}$ was added dropwise and removed after incubation at RT for $30 \mathrm{~min}$. The rabbit anti-human CD-117 antibody (1:100 dilution, ab5505, Abcam) and mouse anti-human tryptase antibody (1:100 dilution, ab2378, Abcam) were added as primary antibodies, and the slides were left at $4^{\circ} \mathrm{C}$ overnight. Subsequently, the slides were retrieved and washed with PBS at RT three times for 5 min each. The Cy3-labeled goat anti-rabbit secondary antibody (1:100 dilution, ab97075, Abcam) and biotin-labeled goat anti-mouse secondary antibody
(1:100 dilution, ab150113, Abcam) were added as secondary antibodies, and the slides were incubated at $37^{\circ} \mathrm{C}$ in the dark for $30 \mathrm{~min}$. Then, the slides were retrieved, washed three times with PBS at RT, counterstained with DAPI, and washed three times again with PBS at RT. Finally, the slides were mounted and preserved using an anti-quenching aqueous mounting agent.

The slides were observed and photographed under a microscope (Figs. 1-3). Ten high-power fields (final magnification $400 \times$ ) were randomly photographed for each slide from the neck, body, and fundus of each gallbladder. The numbers of cells in each field that were CD117-positive $\left(\mathrm{CD} 117^{+}\right)$and tryptase-negative $(-)$ in the cytoplasm and located in the lamina propria or muscularis propria were counted and recorded; the number of cells that were $\mathrm{CD} 117^{+}$and tryptase-positive

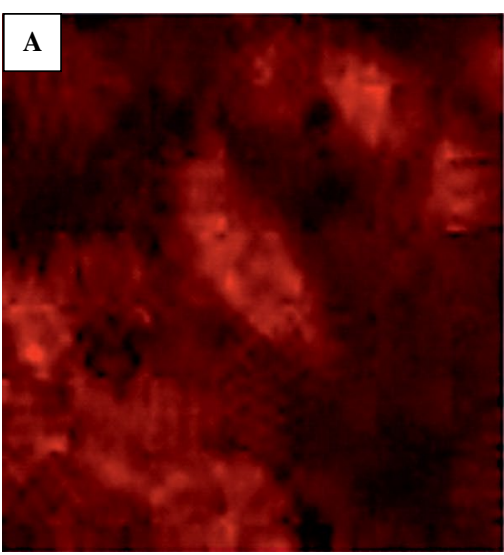

CD117 (+)

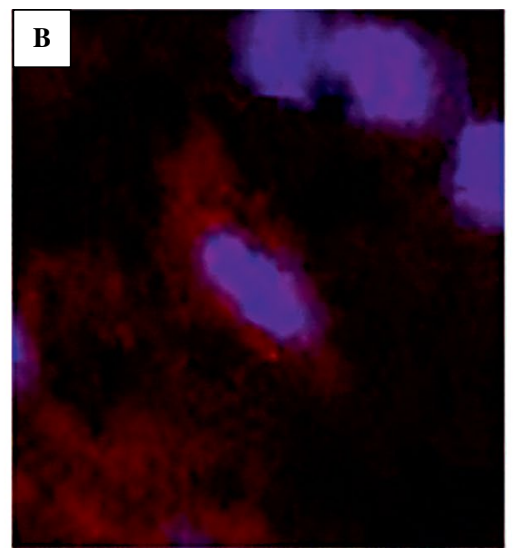

CD117 + Tryptase + DAPI

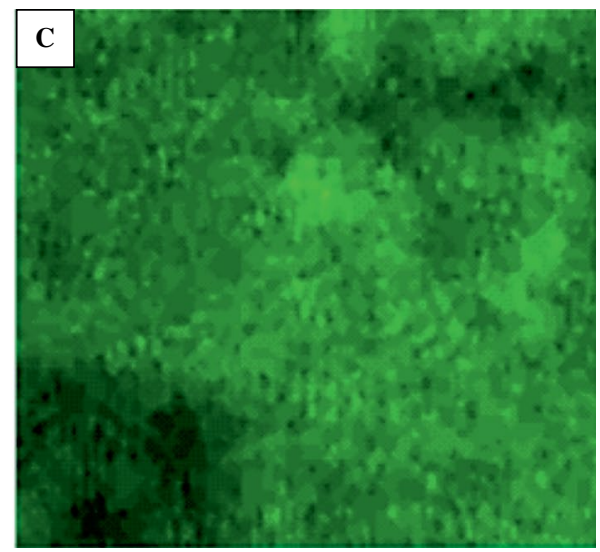

Tryptase (-)

Figure 1. The interstitial Cajal-like cells that were CD117-positive and tryptase-negative in the lamina propria of a patient without cholelithiasis. A. c-kit (C117)-positive cells. B. Merged A and C. C. The same cells were tryptase-negative. Sections were stained by immunofluorescence as described in Methods. DAPI, 4',6-diamidino-2-phenylindole. Magnification: $\times 400$.

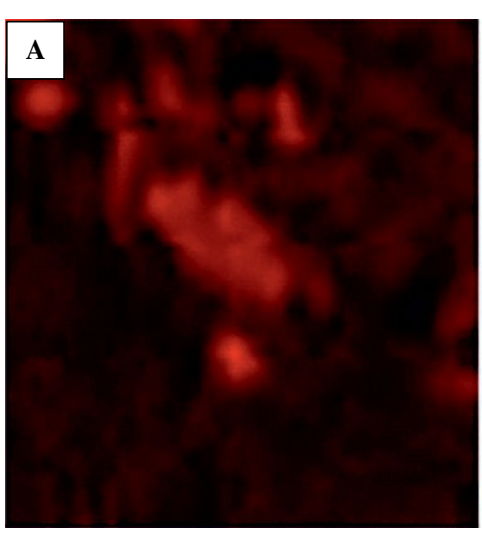

CD117 (+)

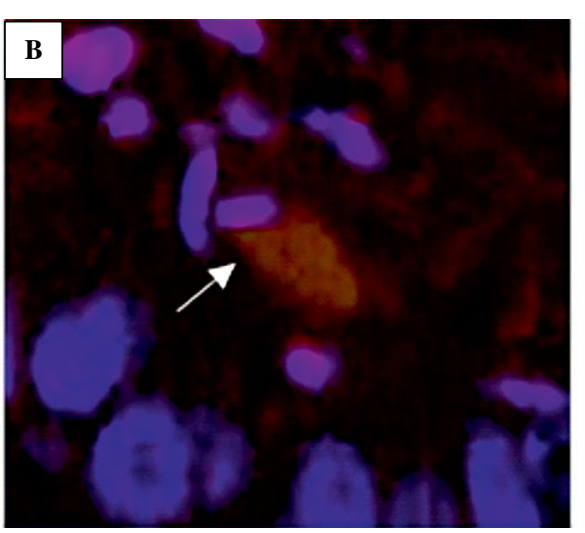

CD117 + Tryptase + DAPI

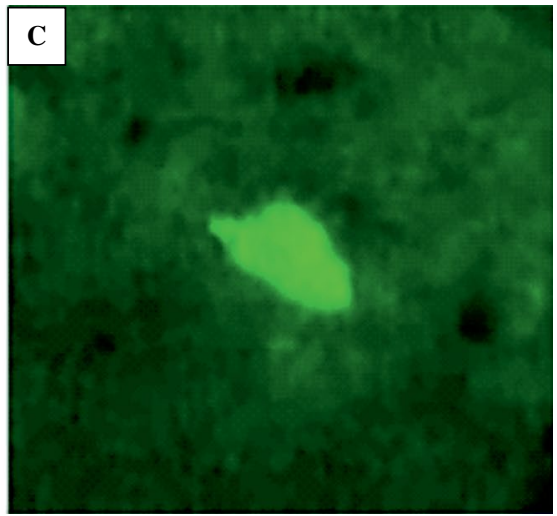

Tryptase (+)

Figure 2. The interstitial Cajal-like cell that is CD117-positive and tryptase-negative in the lamina propria of a patient without

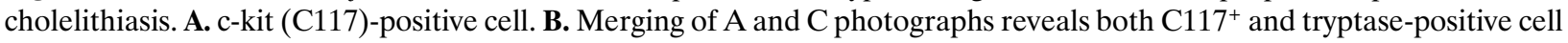
(mast cell). C. The same cell as in (A) is also tryptase-positive. Sections were stained by immunofluorescence as described in Methods. Magnification: $\times 400$. 


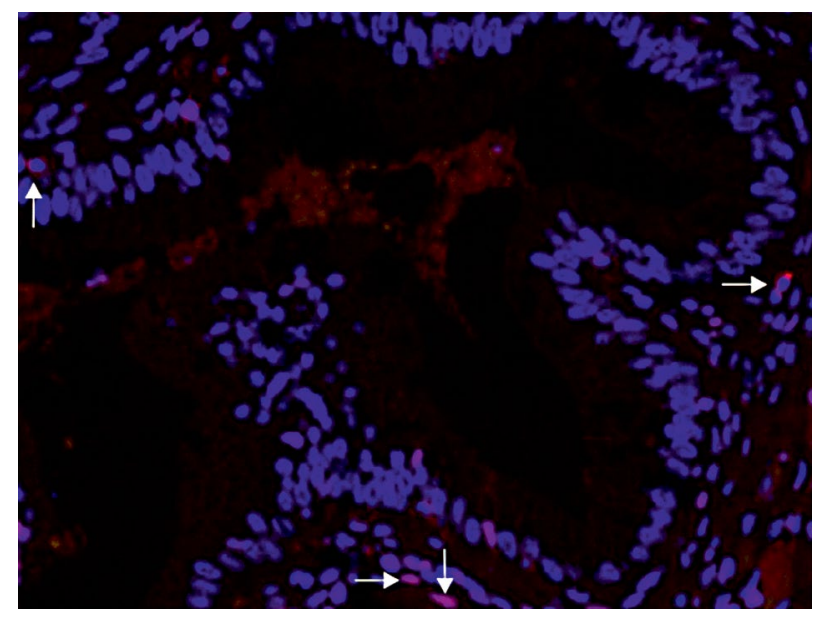

Figure 3. Cross-section of a gallbladder wall from a patient with cholelithiasis shows the presence of a few CD117-positive and tryptase-negative cells (arrows). Magnification: $\times 200$.

$(+)$ were not counted. The recorded data were averaged for statistical analysis.

Statistical analysis. Statistical analysis was performed using SPSS 21.0 (IBM Corp., Armonk, NY, USA). The independent sample t-test was performed to compare the gallbladder contraction rate and number of ICLCs between the cholelithiasis and non-cholelithiasis groups, with $\alpha=0.05$. A scatter plot was drawn using the number of ICLCs as the $\mathrm{X}$-axis and gallbladder contraction rate as the $\mathrm{Y}$-axis, and then Pearson and Spearman correlation analyses were performed. A P-value $<0.05$ indicated that the difference was statistically significant for all the results.

\section{Results}

Based on the aforementioned criteria, 29 patients were screened. Of those, 3 patients were excluded because of incidental discovery of choledocholithiasis, cholelithiasis combined with gallbladder polyps, and a history of gallbladder-preserving lithotripsy. Twenty-six patients were finally included in this study, with 18 patients in the cholelithiasis group and 8 in the non-cholelithiasis group. There were 15 women and 11 men in both groups, and the range of age

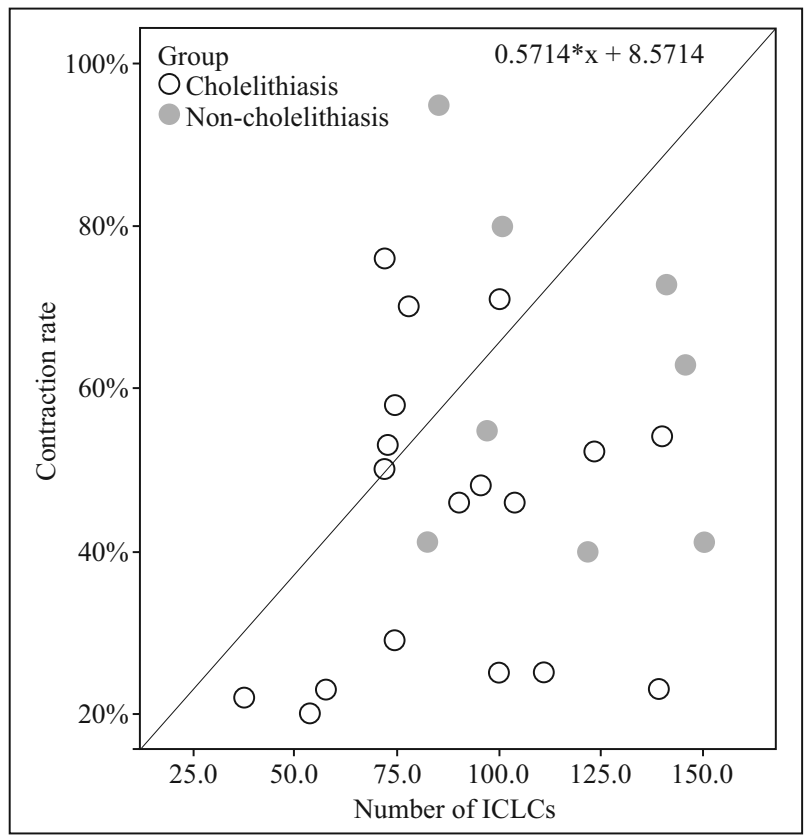

Figure 4. Scatter plot of the number of interstitial Cajal-like cells (ICLCs) and gallbladder contraction rate in patients with and without cholelithiasis.

was 28-77 years (median: both sexes, 60 years; men, 65 ys; women, 57 ys). Twenty-three patients underwent laparoscopic cholecystectomy, 1 patient underwent partial hepatectomy with cholecystectomy, and 2 patients underwent total pancreatectomy.

The independent sample t-test was performed to compare the gallbladder contraction rate between the cholelithiasis and non-cholelithiasis groups, and the difference between the two groups was statistically significant $(43.94 \% \pm 18.50 \%$ vs. $61.00 \% \pm$ $20.50 \%, \mathrm{P}=0.046$; Table 1). The same statistical test was performed to compare the number of ICLCs in the lamina propria between the cholelithiasis and non-cholelithiasis groups, and the difference between the two groups was statistically significant (88.61 \pm 28.22 vs. $115.89 \pm 27.87, \mathrm{P}=0.032$; Table 1 ).

Curve fitting of the scatter plot was performed (Fig. 4), and the Pearson and Spearman correlation analyses revealed no statistically significant correlations $(\mathrm{P}>0.05$, Table 2A, B, C).

Table 1. Comparison of gallbladder contraction rate and the number of ICLCs between patients with and without cholelithiasis

\begin{tabular}{|l|c|c|c|c|c|}
\hline Group & N & Contraction rate, Mean \pm SD & P-value & No. of ICLCs, Mean \pm SD & P-value \\
\hline Cholelithiasis & 18 & $43.9 \% \pm 18.5 \%$ & \multirow{2}{*}{0.046} & $88.611 \pm 28.2207$ & \multirow{2}{*}{0.032} \\
\cline { 1 - 2 } Non-cholelithiasis & 8 & $61.0 \% \pm 20.5 \%$ & & $115.888 \pm 27.8715$ & \\
\hline
\end{tabular}

ICLCs — interstitial Cajal-like cells; no. — number; SD — standard deviation. Independent sample t-test. 
Table 2. Pearson and Spearman correlation analyses of the number of ICLCs and gallbladder contraction rate A) Cholelithiasis Group

\begin{tabular}{|l|c|c|c|c|}
\hline \multicolumn{9}{|c|}{ Spearman correlation coefficient } \\
\hline \multirow{4}{*}{ Spearman Rho } & \multirow{3}{*}{ Contraction rate } & Contraction rate & No. of ICLCs \\
\cline { 3 - 5 } & & Sig (two-tailed) & 1.000 & 0.188 \\
\cline { 3 - 5 } & \multirow{3}{*}{ No. of ICLCs } & N & & 0.455 \\
\cline { 3 - 5 } & & Sig (two-tailed) & 0.455 & 18 \\
\cline { 3 - 5 } & & $\mathrm{N}$ & 18 & 1.000 \\
\hline
\end{tabular}

B) Non-cholelithiasis Group

\begin{tabular}{|c|c|c|c|c|}
\hline \multicolumn{5}{|c|}{ Spearman correlation coefficient } \\
\hline & & & Contraction rate & No. of ICLCs \\
\hline \multirow{6}{*}{ Spearman Rho } & \multirow{3}{*}{ Contraction rate } & Correlation coefficient & 1.000 & -0.180 \\
\hline & & Sig (two-tailed) & & 0.670 \\
\hline & & $\mathrm{N}$ & 8 & 8 \\
\hline & \multirow{3}{*}{ No. of ICLCs } & Correlation coefficient & -0.180 & 1.000 \\
\hline & & Sig (two-tailed) & 0.670 & \\
\hline & & $\mathrm{N}$ & 8 & 8 \\
\hline
\end{tabular}

C) Pearson correlation analysis

\begin{tabular}{|l|c|c|c|}
\hline \multicolumn{5}{|c|}{ Pearson correlation analysis } \\
\hline \multirow{4}{*}{ Contraction rate } & & Contraction rate & No. of ICLCs \\
\hline \multirow{5}{*}{ No. of ICLCs } & Pearson correlation & 1 & 0.168 \\
\cline { 2 - 4 } & Sig (two-tailed) & & 0.411 \\
\cline { 2 - 4 } & $\mathrm{N}$ & 26 & 26 \\
\hline & Pearson correlation & 0.168 & 1 \\
\cline { 2 - 4 } & Sig (two-tailed) & 0.411 & 26 \\
\cline { 2 - 4 } & $\mathrm{N}$ & 26 & \\
\hline
\end{tabular}

For abbreviations, see the legend to Table 1.

\section{Discussion}

ICLCs are a special type of interstitial cell that are widely distributed in various tissues, including the gastrointestinal tract, myocardium, and mammary glands [6-12]. The development and differentiation of these cells are related to their expression of c-kit receptors; hence, without c-kit receptors, ICLCs will degenerate to smooth muscle cells [3]. These cells are also present in biliary tissues. Lavoie et al. [12] showed in tissue extracts that there were synchronous rhythmic $\mathrm{Ca}^{2+}$ flashes in gallbladder smooth muscle cells and ICLCs. After the addition of gap junction uncouplers, the $\mathrm{Ca}^{2+}$ flashes in gallbladder smooth muscle decreased significantly, whereas those of ICLCs remained unchanged. However, after the ad- dition of the c-kit tyrosine kinase inhibitor, imatinib mesylate, the synchronous rhythmic $\mathrm{Ca}^{2+}$ flashes in both tissues were significantly reduced [12]. Thus, the results of this study suggest that ICLCs play a crucial role in the spontaneous rhythmic contraction of gallbladder smooth muscle. Subsequently, some animal experiments have shown that cholecystokinin-A (CCK-A) receptors are expressed in the ICLCs of gallbladder in guinea pigs. Furthermore, in vitro experiments [13] showed that CCK-A can induce significant contractions in gallbladder muscle strips and after removing the ICLCs by exposing the methylene blue-stained muscle strips to light $(532 \mathrm{~nm}$, $50 \mathrm{~mW} / \mathrm{cm}^{2}$ ), the gallbladder muscle strips showed significantly reduced contractions in response to CCK-A. Thus, the study concluded that the contrac- 
tions induced by CCK-A in the gallbladders of guinea pigs may have been mediated by ICLCs [13]. ICLCs have also been observed in the gallbladder tissue of humans [14], and some studies have reported that the number of ICLCs found in gallbladder tissue resected from patients with cholelithiasis was significantly lower than that of patients without cholelithiasis [5]. In an another study [2], it was found that gallbladder contractility, the number of gallbladder ICLCs, and $\mathrm{SCF} / \mathrm{c}$-kit expression level were all significantly lower in patients with cholelithiasis than in those without cholelithiasis. These results imply that the decrease in gallbladder contractility may be associated with the reduced number of gallbladder ICLCs, which in turn may have been caused by the inhibition of the $\mathrm{SCF} / \mathrm{c}$-kit pathway [2].

In the present study, significant differences were found between patients with and without cholelithiasis in terms of the gallbladder contractility assessed by ultrasonography before cholecystectomy and the number of ICLCs. Our findings of lower gallbladder contractility and number of ICLCs in the cholelithiasis group are consistent with those of previous studies [15, 16]. The scatter plot showed that it was highly likely for the number of gallbladder ICLCs to be positively correlated with gallbladder contractility; however, statistical analysis did not reveal a significant correlation. The reason for this finding could be related to numerous factors. Firstly, in the human body, gallbladder contractility is affected by multiple factors. Hence, the number of gallbladder ICLCs may be related to gallbladder contractility, but it cannot determine gallbladder contractility. Possible factors that may be related to gallbladder contractility include but are not limited to the degree of pericholecystic adhesions, thickening of the gallbladder wall [17], number and volume of gallstones in the gallbladder, concentration of cholecystokinin and CCK-A receptors in patients [18], duodenal $\mathrm{pH}$ value, and effects of acupuncture [19]. Secondly, the results of gallbladder contractility based on B-mode ultrasonography may be affected by the subjective judgement of the sonographer which could lead to errors in measuring gallbladder contractility. Additionally, the fatty meal test is only an indirect measure of gallbladder contractility, and ingesting different foods may have an impact on measurements of gallbladder contractility. A previous study suggested that ice candy of the Lundh test meal is ideal for testing gallbladder contractility, and the food item we used differed from that of a common fatty meal test [20]. To avoid the aforementioned errors, it may be necessary to perform in vitro experiments of muscle strip contraction. Experiments of gallbladder muscle strip contraction are widely used in animal studies.
However, in humans, such experiments are subject to many limitations and variables, and they cannot be easily implemented.

Although the present study's results suggest that there is no linear correlation between the number of ICLCs and gallbladder contractility, we cannot exclude the possibility of a non-linear correlation between these two parameters. In addition, as shown by the statistical results and scatter plot, there were significant differences in the gallbladder contractility and number of ICLCs between the cholelithiasis and non-cholelithiasis groups, with the cholelithiasis group showing significantly reduced gallbladder contractility and number of ICLCs compared to the non-cholelithiasis group. These findings imply that we should still consider that gallbladder contractility may be related to the number of gallbladder ICLCs. However, the nature and extent of this relationship(s) will require future investigation, and the construction of a mathematical model may involve multiple related factors.

Our study has several limitations. First, our study included a small number of cases. If we had at least 60 cases in this study, we would have achieved a more precise result. Second, interpretation of the results of ultrasonography is subjective. Third, the in vitro measurements of muscle-strip contraction may have been better than the Lundh test for evaluating the contraction rate of the gallbladder. Despite these limitations, we believe that the significant differences in the in vivo gallbladder contractility and the number of gallbladder ICLCs between cholelithiasis and non-cholelithiasis patients suggest that factors other than solely the number of gallbladder ICLCs affect gallbladder contractility in patients with cholelithiasis.

\section{Acknowledgments}

This study was supported by Beijing Hospital, National Center of Gerontology. We also thank Jiamay Biotech, Co., Ltd. for providing the reagent and technology support.

\section{References}

1. Stinton LM, Shaffer EA. Epidemiology of gallbladder disease: cholelithiasis and cancer. Gut Liver. 2012; 6(2): 172-187, doi: 10.5009/gnl.2012.6.2.172, indexed in Pubmed: 22570746.

2. Tan YY, Ji ZL, Zhao G, et al. Decreased SCF/c-kit signaling pathway contributes to loss of interstitial cells of Cajal in gallstone disease. Int J Clin Exp Med. 2014; 7(11): 4099-4106, indexed in Pubmed: 25550919.

3. Pasternak A, Szura M, Gil K, et al. Interstitial cells of Cajal - systematic review. Folia Morphol (Warsz). 2016; 75(3): 281-286, doi: 10.5603/FM.a2016.0002, indexed in Pubmed: 26806433.

4. Xu D, Yu BP, Luo HS, et al. Control of gallbladder contractions by cholecystokinin through cholecystokinin-A receptors 
on gallbladder interstitial cells of Cajal. World J Gastroenterol. 2008; 14(18): 2882-2887, doi: 10.3748/wjg.14.2882, indexed in Pubmed: 18473415.

5. Pasternak A, Gil K, Matyja A, et al. Loss of gallbladder interstitial Cajal-like cells in patients with cholelithiasis. Neurogastroenterol Motil. 2013; 25(1): e17-e24, doi: 10.1111/ nmo.12037, indexed in Pubmed: 23121223.

6. Komuro T. Structure and organization of interstitial cells of Cajal in the gastrointestinal tract. J Physiol. 2006; 576(Pt 3): 653-658, doi: 10.1113/jphysiol.2006.116624, indexed in $\mathrm{Pu}-$ bmed: 16916909.

7. Popescu LM, Hinescu ME, Ionescu N, et al. Interstitial cells of Cajal in pancreas. J Cell Mol Med. 2005; 9(1): 169-190, doi: 10.1111/j.1582-4934.2005.tb00347.x, indexed in Pubmed: 15784175 .

8. Hinescu ME, Gherghiceanu M, Mandache E, et al. Interstitial Cajal-like cells (ICLC) in atrial myocardium: ultrastructural and immunohistochemical characterization. Journal of Cellular and Molecular Medicine. 2006; 10(1): 243-257, doi: 10.1111/j.1582-4934.2006.tb00306.x.

9. Shafik A, El-Sibai O, Shafik I, et al. Immunohistochemical identification of the pacemaker cajal cells in the normal human vagina. Arch Gynecol Obstet. 2005; 272(1): 13-16, doi: 10.1007/s00404-005-0725-3, indexed in Pubmed: 15834581.

10. Radu E, Regalia T, Ceafalan L, et al. Cajal-type cells from human mammary gland stroma: phenotype characteristics in cell culture. J Cell Mol Med. 2005; 9(3): 748-752, doi: 10.1111/ j.1582-4934.2005.tb00509.x, indexed in Pubmed: 16202226.

11. van der AA F, Roskams T, Blyweert W, et al. Identification of kit positive cells in the human urinary tract. J Urol. 2004; 171(6 Pt 1): 2492-2496, doi: 10.1097/01.ju.0000125097.25475.17, indexed in Pubmed: 15126883.

12. Lavoie B, Balemba OB, Nelson MT, et al. Morphological and physiological evidence for interstitial cell of Cajal-like cells in the guinea pig gallbladder. J Physiol. 2007; 579(Pt 2): 487-501, doi: 10.1113/jphysiol.2006.122861, indexed in Pubmed: 17204499.

13. Xu D, Yu BP, Luo HS, et al. Control of gallbladder contractions by cholecystokinin through cholecystokinin-A receptors on gallbladder interstitial cells of Cajal. World J Gastroenterol. 2008; 14(18): 2882-2887, doi: 10.3748/wjg.14.2882, indexed in Pubmed: 18473415 .

14. Hinescu ME, Ardeleanu C, Gherghiceanu M, et al. Interstitial Cajal-like cells in human gallbladder. J Mol Histol. 2007; 38(4): 275-284, doi: 10.1007/s10735-007-9099-0, indexed in Pubmed: 17541711.

15. Runyu $\mathrm{D}$, Jingyong $\mathrm{X}$. The relationship between gallstone formation and the interstitial cell of Cajal like cell in gallbladder. Chinese J Med. 2017; 52: 25-27.

16. Ying F, Shouhua W, Zhenhua Y, et al. The change of slow wave and tension of muscle strip from gallbladder of guinea pig after wiping off interstitial cell of Cajal like cell. Chin J Exp. 2012; 2: 850-850.

17. Jing S, Weixu C, Xia W, et al. The role and mechanism of gallbladder wall thickening in gallstone formation. J Xuzhou Med Univ. 2017; 37: 763-765.

18. Ruixin C, Ming Ya, Zhiwei W, et al. Relationship between CCK-AR mRNA of gallbladder wall and plasma CCK-8 level and gallbladder emptying function in gallstone patients. Shandong Med J. 2006; 36: 1-2.

19. Yanbin Z, Ziyang C, Peijin L, et al. Clinical research of ultrasound in monitoring gallbladder constriction after acupuncture and lipoid-food test in diagnosis of cholecystitis. Chinese Imaging J Integr Tradit Western Med. 2010; 8: 193-195.

20. Tairan S, Kai Z, Yubao S, et al. The application values of real-time ultrasound and the ice candy of LUNDH test meal in checking the gallbladder contractive function. Chin J Med Ultrasound. 2011; 8: 2558-2563.

Submitted: 29 August, 2018

Accepted after reviews: 19 June, 2019

Available as AoP: 24 June, 2019 